\title{
A new additional energy source for tornadoes
}

\author{
Francesco Meneguzzo $^{1, ~ *, ~ Y u r i y ~ A l e x e e v i c h ~ B a u r o v ~}{ }^{2,3}$, Lorenzo Albanese ${ }^{1}$ \\ ${ }^{1}$ National Research Council - Institute of Biometeorology, 8, Via G. Caproni, I-50145Firenze, Italy \\ ${ }^{2}$ Closed Joint Stock Company Research Institute of Cosmic Physics, 141070, Moscow Region, Pionerskaya, 4, Korolyov, Russia \\ ${ }^{3}$ Hotwater Srl, Via Gioberti, 15, I-56024 San Miniato (PI), Italy
}

\section{Emailaddress:}

f.meneguzzo@ibimet.cnr.it (F. Meneguzzo),baurov@mail.ru (Y. A. Baurov), 1.albanese@ibimet.cnr.it (L. Albanese)

\section{To cite this article:}

Francesco Meneguzzo, Yuriy Alexeevich Baurov, Lorenzo Albanese. A New Additional Energy Source for Tornadoes. American Journal of Astronomy and Astrophysics. Special Issue: Global Anisotropy, Theory of Byuon, New Force, New Power System, Propulsion, Space Flights. Vol. 2, No. 6-1, 2014, pp. 32-39. doi: 10.11648/j.ajaa.s.2014020601.15

\begin{abstract}
Following earlier research aimed at detecting evidence of a connection between selected very powerful tornadoes and the global anisotropy of the physical space representing the foundation of the theory of byuons, allegedly realized by means of a positive feedback between the tornado updraft and the cosmological vector representing the global anisotropy, this article extends the analysis to a much larger database of tornadic events occurring in the continental U.S. in order to detect the fingerprint of the new proposed physical processes on the overall statistics. While the theoretical foundations of the onset and development of tornadoes are well established, some work remains to be done with particular reference to the upper tail of the energy distribution of tornadoes, where the energy developed by a single event can be as huge as $10^{16} \mathrm{~J}$, or the equivalent of 2 Mton of TNT. In this range, additional mechanisms of energy supply in the process of development of a tornado are proposed and their fingerprint on the overall statistics are looked for. Although further analyses over different geographical areas, especially in the southern hemisphere, would be needed in order to provide a really conclusive proof, convincing evidence accumulates and points to the reality of the new proposed physical processes.
\end{abstract}

Keywords: Tornado, Fujita Scale, Theory of Byuons, New Energy, Global Anisotropy of the Physical Space

\section{Introduction}

The nature of the origin of tornadoes, especially those developing into violent ones (F3 to F5 on the Fujita scale) has always been of great interest for researchers, since the destructive energy of such tornadoes is comparable with the energy of nuclear explosions, i.e. on the order of $20 \mathrm{kton}$ of TNT, or $10^{14} \mathrm{~J}[1]$.

A great deal of theoretical and modeling work was performed regarding the onset and time evolution of tornadoes[1-3], as well as very effective conceptual models were developed, as summarized by Kufa and Snow [4].

Numerical simulations of real tornadoes sometimes suffer from significant underestimation of their intensity, even using the most advanced mesoscale or local scale meteorological models, such as the state of the art WRF-NMM model used in Litta et al. [5]: their F3 tornado occurred in India was simulated as a much weaker F0 one (peak wind speed around $20 \mathrm{~ms}^{-1}$ against the observed $70 \mathrm{~ms}^{-1}$ ), that is most of its real energy was not caught by the simulation. Nevertheless, such models have been successfully used for the simulation and prediction of the local atmospheric "ingredients" leading to tornadoes [6].

Besides the maximum intensity based on damages (Fujita classification) which is heavily dependent upon the strength of the winds inside the tornado, recently the path length and the path width of a tornado were included in a more comprehensive and physically consistent analysis of its overall energy in the effort to derive a power law describing the temporal behavior of tornadoes analogously to Earthquakes [7].

Some additional contribution could still be useful to explain the source of the huge energy of the most powerful tornadic events in the course of their development in time; this is the subject of the present work and will be performed in the frame of a known physical theory which application to the field of atmospheric sciences is quite recent [8].

The paper is organized as follows: Section 2 describes the tornado dataset used and its processing in order to derive the needed quantities for the subsequent analysis; in Section 3 theoretical insights are proposed about the byuon theory and the global anisotropy of the physical space (Section 3.1.), as 
well as the role of such anisotropy for the additional energy supply to tornadoes (Section 3.2.); Section 4 shows the results of the verification of the processes explained in Section 3.2. as well as the respective discussion. The conclusions and the perspectives for further work are set out in Section 5, the last of this article.

\section{Materials and Methods}

In the search for clues supporting the main hypothesis described in Section 3.2, tornado data including date, time, peak intensity, geographical coordinates of tornado's touchdown and its path length and width are needed. Tornado reporting worldwide lags very much behind the quality as well as the continuous and homogeneous coverage used in the U.S.A., therefore all the data used in this article refer to that Country.

The data were drawn from the Storm Prediction Center (SPC) database [9], in the period 1954 to 2013; as a major improvement over previous research on the same subject [8], the whole SPC dataset was considered, not only individual selected events, even if the analysis of significant tornado outbreaks carried out in that article was especially significant and convincing [8].

Fig. 1 shows the geographical area, covering part of the continental U.S., considered for the tornado statistics: latitude $30^{\circ} \mathrm{N}$ to $50^{\circ} \mathrm{N}$, longitude $105^{\circ} \mathrm{W}$ to $85^{\circ} \mathrm{W}$. This choice was motivated by the relative homogeneity of the synoptic ingredients leading to tornadic outbreaks in the study area, i.e. moist inflow from the Gulf of Mexico and vertical wind shear in turn connected with the mid-latitude jet stream [6].

In order to homogenize the dataset and obtain all the needed physical quantities, the original SPC data were processed as follows.

Following to the information about the shift of reporting from tornado mean width till the year 1994 to maximum width afterwards [9], the maximum width reported during 1995 to 2013 was turned back to the mean width after multiplying by a conversion factor which value was computed separately for any Fujita intensity class, as shown in Table 1.

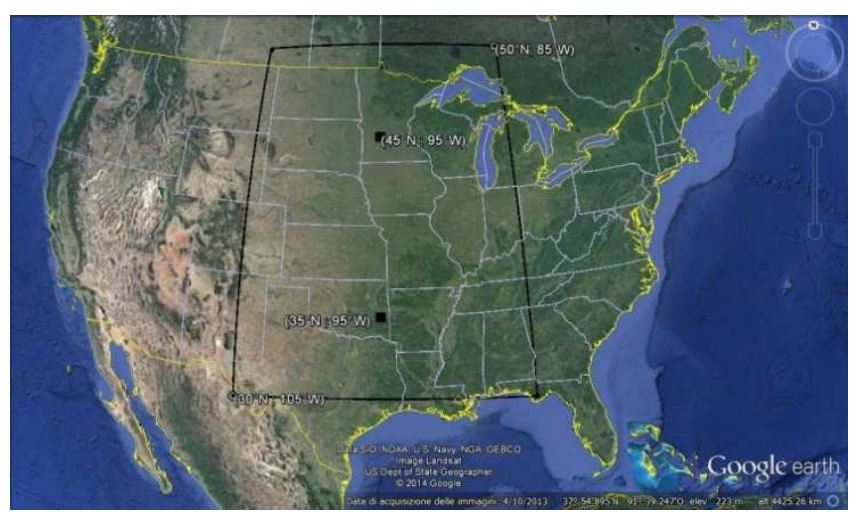

Fig. 1. Geographical area considered for the tornado statistics.
Table 1. Climatological mean and maximum width of tornadoes and their ratio for any intensity class.

\begin{tabular}{lcccccc}
\hline & \multicolumn{6}{c}{ Fujita intensity class } \\
\cline { 2 - 7 } & F0 & F1 & F2 & F3 & F4 & F5 \\
\hline Mean Width (m) 1954 - 1994 & 29 & 63 & 121 & 262 & 452 & 562 \\
Maximum Width (m) 1995 - 2013 & 45 & 131 & 289 & 582 & 848 & 1,425 \\
Mean to Maximum width ratio & 0.64 & 0.48 & 0.42 & 0.45 & 0.53 & 0.39 \\
\hline
\end{tabular}

The average height $(h)$ of a tornado was estimated, following Kurgansky [10], assuming its proportionality to the mean width $(d): h / d=\gamma=$ constant, where $\gamma \sim 10-30$; the central value $\gamma=20$ was used. Moreover, minimum and maximum values for the tornado height were set at $1,000 \mathrm{~m}$ and $9,000 \mathrm{~m}$, respectively [10].

As a further major improvement over previous work on the subject of this article [8], the total atmospheric moment (energy) was computed for each tornado event based upon the works by Schielicke and Névir [7,11]; having estimated the tornado height too, the following expression for the tornado moment was used:

$$
M=W \cdot H \cdot L \cdot \rho \cdot e
$$

where $M$ is the moment, $W$ is the tornado mean width, $H$ is the tornado height, $L$ is the tornado path length, $\rho$ is the average air density (approximated to $1 \mathrm{kgm}^{-3}$ ), $e$ is the tornado mass-specific kinetic energy, estimated as $e=$ $\langle v(F)\rangle^{2} / 2$ where $\langle v(F)\rangle$ is the mean value of the wind speed concerning a specific Fujita class and is calculated by the relation of Fujita intensity scale and velocity: $\langle v(F)\rangle=$ $6.30 \mathrm{~ms}^{-1} \cdot(F+2.5)^{3 / 2}$ where $F$ is the specific Fujita class (F0 to F5) [7]. All the units are expressed in MKS physical unit system, therefore the unit of momentum is Joule (J).

After discarding the events lacking the needed details, the total number of tornadoes occurred in the study area was 40,061 out of which 18,370 F0 events, 12,916 F1, 6,388 F2, $1,869 \mathrm{~F} 3,463 \mathrm{~F} 4$ and $55 \mathrm{~F} 5$ events.

Fig. 2 shows the frequency distribution of the moment over the whole dataset of 40,061 considered tornado events (Fig. 2a) and limited to values exceeding the threshold of $10^{13} \mathrm{~J}$ (Fig. 2b); few events appear to have moment on the order of $10^{16} \mathrm{~J}$, or 2 Mton of TNT.

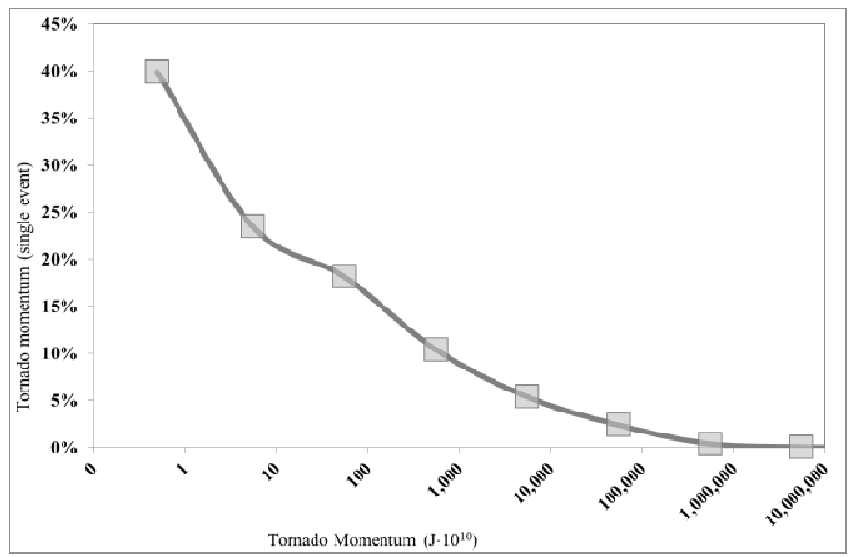

(a) 


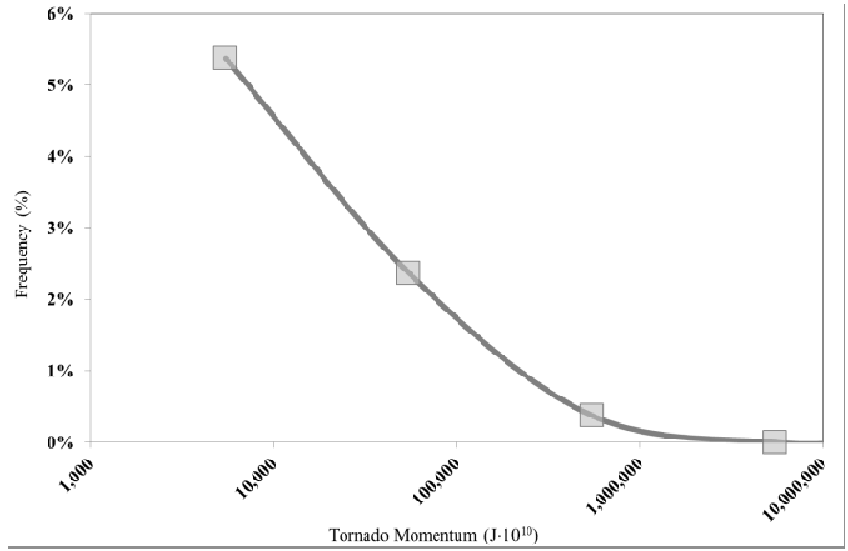

(b)

Fig. 2. Frequency distribution of the tornado moment: whole data set (a) and over the threshold of $10^{13} \mathrm{~J}(\mathrm{~b})$.

Turning to a class by class analysis, limited to F3 to F5 events, Fig. 3 shows the average moment (Fig. 3a), path length (Fig. 3b) and path width (Fig. 3c) over the whole dataset under study.

The average moment of F5 events exceeds that of F4 events by about 4 times, and the moment of F3 by more than an order of magnitude (Fig. 3a).

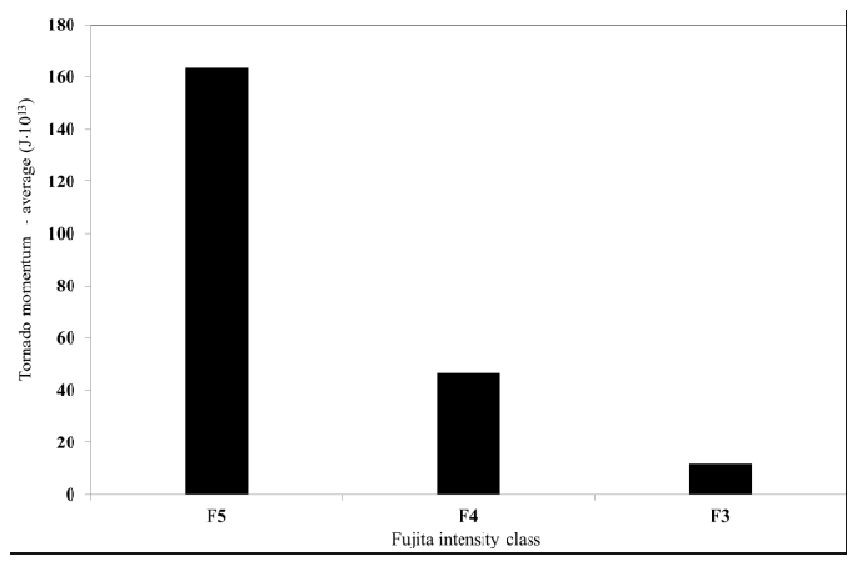

(a)

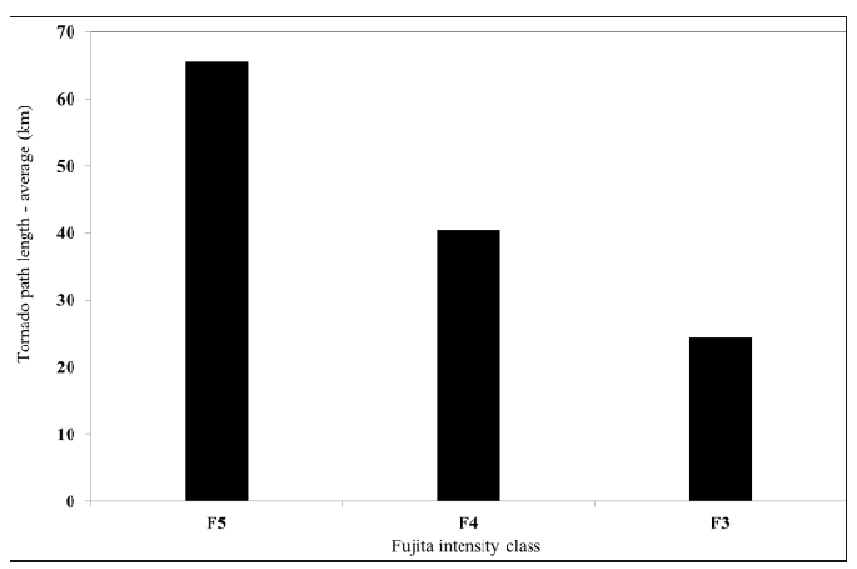

(b)

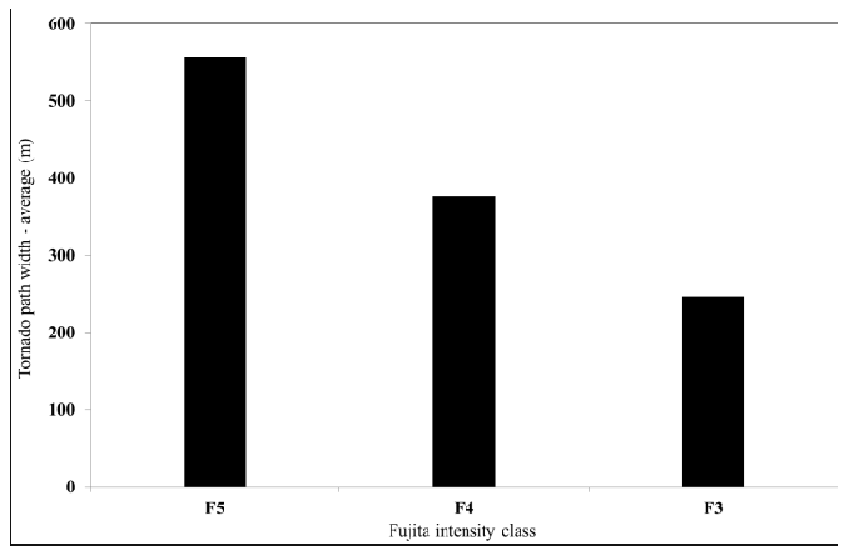

(c)

Fig. 3. Average moment (a), path length (b) and path width (c) for tornado events falling into the F5, F4 and F3 Fujita classes.

It arises that the large differences in the average tornado moment, computed according to Eq. (1), is due not only to the different mean values of the wind speed, which are classspecific, and therefore to the different mass-specific kinetic energy, because the path length and width (and consequently height) contribute significantly to further differentiate the overall average moment.

This evidence points to a significant relationship between rotational velocity, width and lifetime (path length) of tornadoes, supporting the idea of common underlying mechanism working at least for the most energetic events, which will be discussed in the next Sections.

\section{Theoretical Insights}

\subsection{The Global Anisotropy of the Physical Space and the Byuon Theory}

In $[12,13]$ the earliest experiments are described where a fundamental anisotropy of the physical space as well as a new non-gauge interaction, different from the four known ones (strong, weak, electromagnetic, and gravitational), were first detected. Besides the lack of gauge invariance, an important feature of this new interaction is just its anisotropy that arises in a wide range of sizes, from the weak interactions, i.e. $10^{-19} \mathrm{~m} \mathrm{[14]}$, up to the size of our Galaxy, i.e. $10^{21} \mathrm{~m}[15]$, and more [16].

The physical nature of the new force is explained by the byuon theory, a non-gauge theory of the formation of the physical space and the world of elementary particles from some unobservable objects called "byuons" [17-19].

According to the byuon theory [17-20] the potentials of physical fields can act upon the process of mass formation of the elementary particles because a fraction of such mass, associated with the formation of their inner space, is proportional to the modulus of a summary potential $A_{\Sigma}$, i.e. the sum of the potentials of all known force fields calculated using the energy relation expressed by Eq. (3.1.) in Baurov and Malov [21]. Such summary potential cannot exceed, by 
magnitude, the modulus of the cosmological vectorial potential $\mathrm{A}_{\mathrm{g}}$, a new fundamental constant having absolute value $\left|\mathrm{A}_{\mathrm{g}}\right| \approx 1.9 \cdot 10^{5} \mathrm{~T} \cdot \mathrm{m}$.

As the result of the action of the field potentials (decreasing $\left|\mathrm{A}_{\Sigma}\right|$ ), each particle gains an energy $\Delta \mathrm{m} \cdot \mathrm{c}^{2}$ that corresponds to a new force of nature throwing substance out of the region with the weakened $A_{\Sigma}$. Experimental investigations with the use of gravimeters and magnets $[17,18]$, and plasma systems $[17,18,22]$, as well as the measurements of changes in the $\beta$-decay rate of radioactive elements $[14,17,18,23]$, that were later confirmed by independent researches [24], have shown that the substance is ejected from the region with the weakened $\mathrm{A}_{\Sigma}$ along a cone with an angular opening about $100^{\circ}$ around the vector $\mathrm{A}_{\mathrm{g}}$ determining the global anisotropy of the physical space.

In [20] the following astronomical coordinates in the second equatorial system were determined for the vector $\mathbf{A}_{\mathbf{g}}$ and therefore for the global anisotropy of the physical space: $\alpha \approx 300^{\circ} \pm 10^{\circ}, \quad \delta \approx 36^{\circ} \pm 10^{\circ}$, where $\alpha$ is the right ascension and $\delta$ is the declination, as the most exact results so far.

Fig. 4 shows the direction of the cosmological vector potential $A_{g}$ projected onto the Earth's orbital plane, representing the direction of the global anisotropy of the physical space.

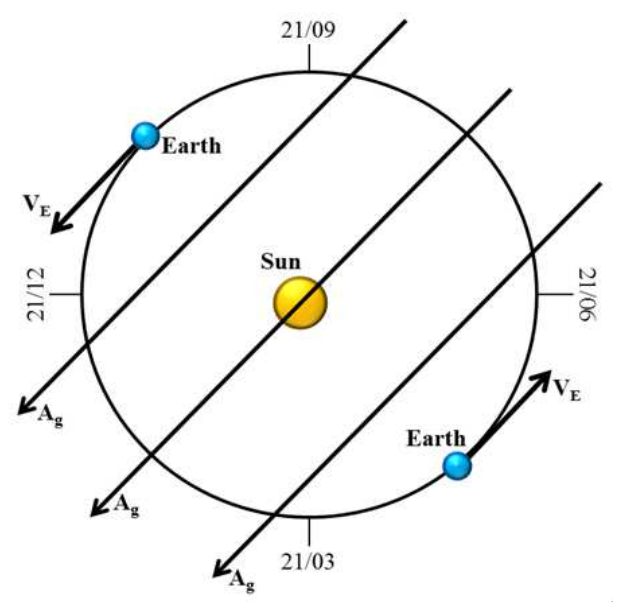

Fig. 4. Projection of the cosmological vector potential $A_{g}$ onto a simplified Earth's orbital plane; $V_{E}$ is the Earth's instantaneous velocity vector.

Fig. 5 shows the dependence of the altitude of the cosmological vector $A_{g}$ on the local solar time, or Central Standard Time (CST) across the year, every two months starting on mid-February, at two sites with coordinates $\left(35^{\circ} \mathrm{N} ; 95^{\circ} \mathrm{W}\right)$ (Fig. 5a) and $\left(45^{\circ} \mathrm{N} ; 95^{\circ} \mathrm{W}\right)$ (Fig. $\left.5 \mathrm{~b}\right)$, as shown in Fig. 1 (year 2001 is considered, with the interannual change during the study period being negligibly small). Troughs and peaks move backward in time by two hours per month; the $\mathrm{A}_{\mathrm{g}}$ altitude range is apparently larger at $35^{\circ} \mathrm{N}$ (approximately $-20^{\circ}$ to $87^{\circ}$ ) than at $45^{\circ} \mathrm{N}\left(-10^{\circ}\right.$ to $\left.81^{\circ}\right)$, therefore the time of residence of the altitude itself around $0^{\circ}$ (i.e. $\mathrm{A}_{\mathrm{g}}$ lying around the local horizontal plane), decreases with increasing latitude.

The analysis of a long run of experiments has shown that the new force has a nonlinear and nonlocal character and can be represented as a complex series in terms of changes of the summary potential $\mathrm{A}_{\Sigma}$ as per Eq.(4) in Baurov [21] (see also $[13,17,18])$.

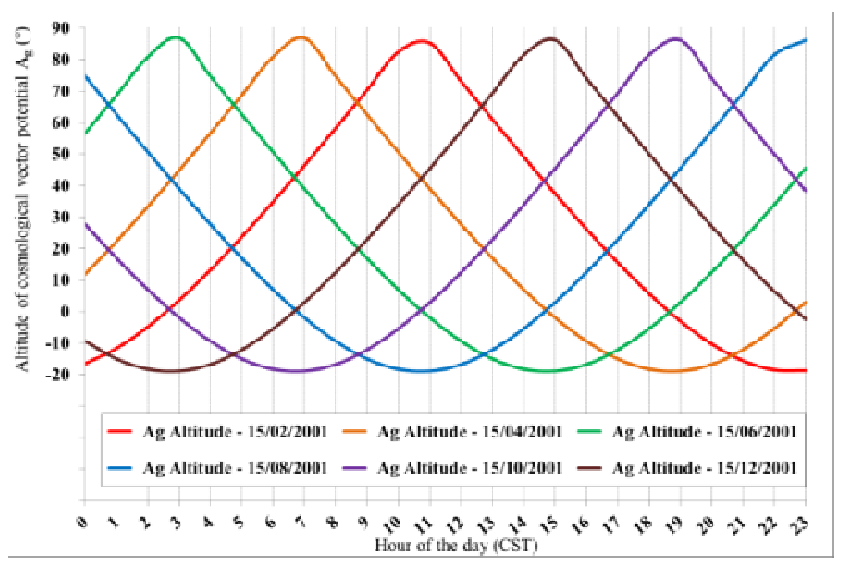

(a)

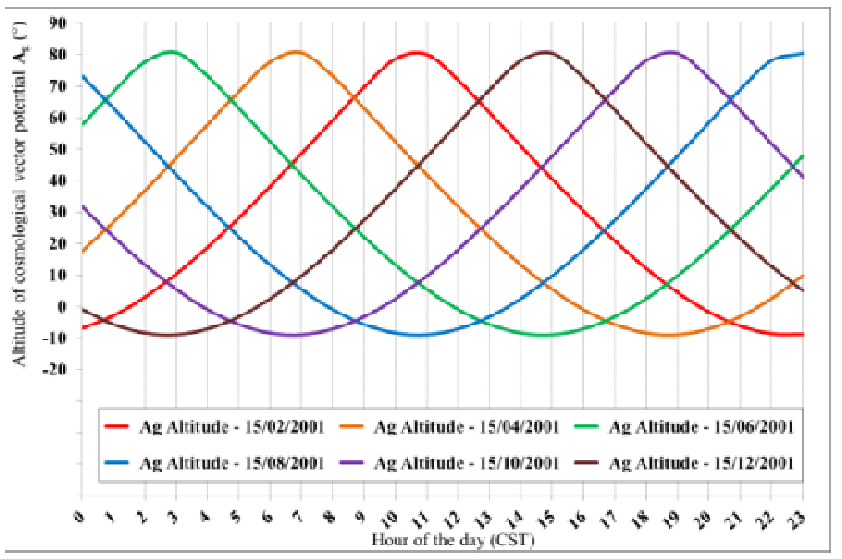

(b)

Fig. 5. Dependence of the altitude of the cosmological vector potential $A_{g}$ on the local solar time $(\mathrm{CST})$ at sites with coordinates $\left(35^{\circ} \mathrm{N} ; 95^{\circ} \mathrm{W}\right)(\mathrm{a})$ and $\left(45^{\circ} \mathrm{N} ; 95^{\circ} \mathrm{W}\right)$.

The first term of the series is the following:

$$
F=2 N m_{v} c^{2} \lambda_{1}^{2} \Delta A_{\Sigma}\left[\Delta\left(\Delta A_{\Sigma}\right) / \Delta x\right]
$$

where $N$ is the number of stable particles (electrons, protons, and neutrons) in the test body, $\Delta A_{\Sigma}$ is the difference in changes of the summary potential $\mathrm{A}_{\Sigma}$ at the location points of a test body and sensor element, $\Delta\left(\Delta A_{\Sigma}\right) / \Delta x$ is the gradient in space of the difference potentials $\Delta A_{\Sigma} ; x$ is the length of an arc of a circle for experiments with solenoids, therefore a space coordinate; $2 m_{v} c^{2}=33 \mathrm{eV} ; \lambda_{1}=10^{-6}(\mathrm{~T} \cdot \mathrm{m})^{-1}$ is the first coefficient of the series.

The fundamental research of the global anisotropy of physical space along with the basics of the byuon theory based upon such anisotropy is summarized in Baurov [20] which includes all the relevant bibliography.

The revealed anisotropic properties of the physical space were found to affect as well very powerful geophysical processes such as the strongest earthquakes, offering a chance for a deeper understanding and even a reference 
framework for a possible prediction in future [25].

The following few consequences of the byuon theory are most important in the context of this article:

- The potential of the Earth's gravitational field is always negative and decreases in absolute value with height from the local surface, therefore as per Eq. (2) a particle rising from the surface with high enough speed, experiencing a substantial rate of decrease of the gravitational potential, is subjected to an upward force towards higher values of the summary potential $\mathrm{A}_{\Sigma}$.

- Quantum states of the byuons interact in regions of weakened summary potential to minimize their potential energy, creating the fundamental onedimensional physical space along with rotation of the material substance around an axis perpendicular to the cosmological vector potential $\mathbf{A}_{\mathbf{g}}$, which arises from the residues of such minimization [17-19].

- $\quad$ More precisely, most of the residual potential energy of interaction of byuons in the fundamental onedimensional space created by the byuons themselves converts into the spin of primary particles, such as the electron, any lepton, etc., as well as into its mass; according to the byuon theory, any natural rotation such as the rotation of planets, stars, galaxies and the expected rotation of the Universe, arises from this mechanism of minimization of the potential energy of interaction of byuons [17-19].

\subsection{Tornadoes and the Global Anisotropy of the Physical Space}

Whatever the processed leading to the onset of a tornado, afterwards it can further accumulate energy along its path by means of well understood processes [2,3]; two further concurrent processes are proposed here, which are thought to sustain or reinforce a tornado during its development.

The first process stems from the consideration, already expressed in Section 3.1., that the reduction of the summary potential $A_{\Sigma}$ by means of the Earth's gravitational potential, that is always negative, is stronger near the Earth's surface than at any height along the local vertical axis; therefore, according to Eq. (2) the flow in an updraft is further accelerated at the expense of the gravitational potential in the direction of the increasing summary potential, which coincides with the direction to the local zenith.

Such process appears similar to the one described in [18,26,27]: during the motion of a liquid substance powered by a centrifugal pump, in a closed circuit having ascending and descending sections (height between $2.6 \mathrm{~m}$ and $4 \mathrm{~m}$ ), excess heat is generated at the level of at least $30 \%$ more than the input energy, which can't be explained from the perspective of standard calibrated physical models. Of course, the stronger the updraft, the greater the acceleration because the uprising particles experience a faster change (decrease) of the summary potential.

The second process is likely even more important and specific to tornadoes. Recalling Section 3.1., any natural rotation arises from the mechanism of minimization of the potential energy of interaction of byuons in their fundamental one-dimensional space [19].

The latter applies as well to a tornado that can gain a fraction of energy of its rotational motion from the minimization of the potential energy of interacting byuons in regions with summary potential weakened by means of the Earth's gravitational potential; provided that the cosmological vector potential lies around the horizontal plane tangent to the local Earth's surface, this process results in the generation of further rotation around the vertical axis of any particle while rising in the violent updraft associated to the tornado, thus further contributing to the energy and lifetime of the tornado itself.

Fig. 6 shows a very simplified scheme of the proposed processes contributing to the energy of a tornado system.

It should be noted that a small deviation of $\mathrm{A}_{\mathrm{g}}$ with regards to the horizontal plane, represented by the angle $\beta$ in Fig. 6 , could be even more favorable for the energy supply to the tornado because the vertical wind shear, which is a necessary synoptic ingredient for its onset, effectively tilts the tornado axis from the vertical; as a first guess, the optimal value for the angle $\beta$ could be in the range $10^{\circ}$ to $20^{\circ}$, positive (upward) or negative (downward).

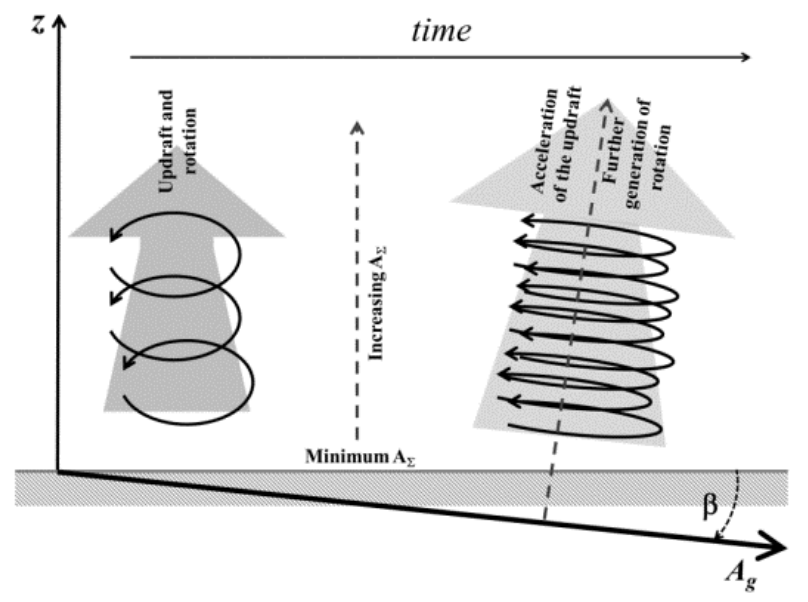

Fig. 6. Very simplified scheme of the proposed processes contributing to the energy of a tornado system: $A_{g}$ is the cosmological vector potential and $\beta$ is its altitude with regards to the local Earth's surface (shaded in the picture).

As a result, the new proposed mechanisms could help to discriminate situations favorable for the further accumulation of energy into a tornado and therefore its development into a high intensity and/or long lifetime or path system.

\section{Results and Discussion}

Although the physical mechanisms leading to the onset and development of tornadoes have been extensively studied and are currently well known, as pointed out in Section 1, earlier analysis suggests that the herein proposed further energy supply mechanisms, based upon the theory of byuons, arise with convincing evidence at least when dealing with the most violent and energetic events. 
With a substantially larger dataset such as the one available for this research, the advanced hypotheses should leave their fingerprint in the overall statistics of the most violent tornadoes.

Fig. 7(a-f) shows the frequency distribution of the total tornado moment along with the significant features of individual events, i.e. moment (Fig. 7a and Fig. 7b), path length (Fig. 7c and Fig. 7d) and mean path width (Fig. 7e and Fig. 7f), as functions of the altitude of the cosmological vector potential $\mathbf{A}_{\mathbf{g}}$ in intervals of $10^{\circ}$, separately for the Fujita classes F5 (Fig. 7a, Fig. 7c and Fig. 7e) and F4 (Fig. 7b, Fig. 7d and Fig. 7f). The range of altitudes of $\mathbf{A}_{\mathbf{g}}$ occurred during the tornado lifetime is shown only for events with moment greater than $2 \cdot 10^{15} \mathrm{~J}$.

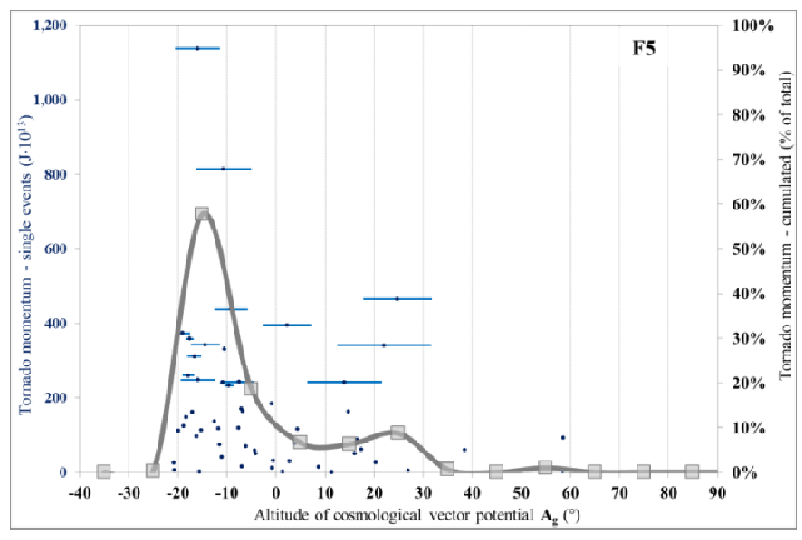

(a)

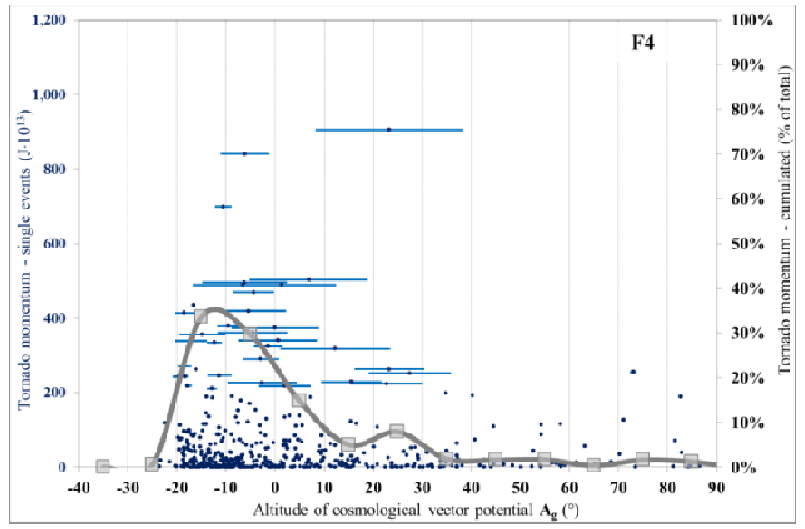

(b)

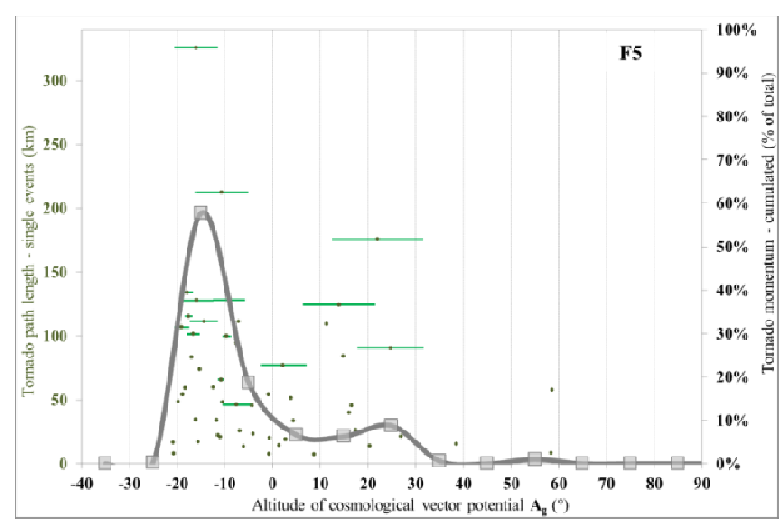

(c)

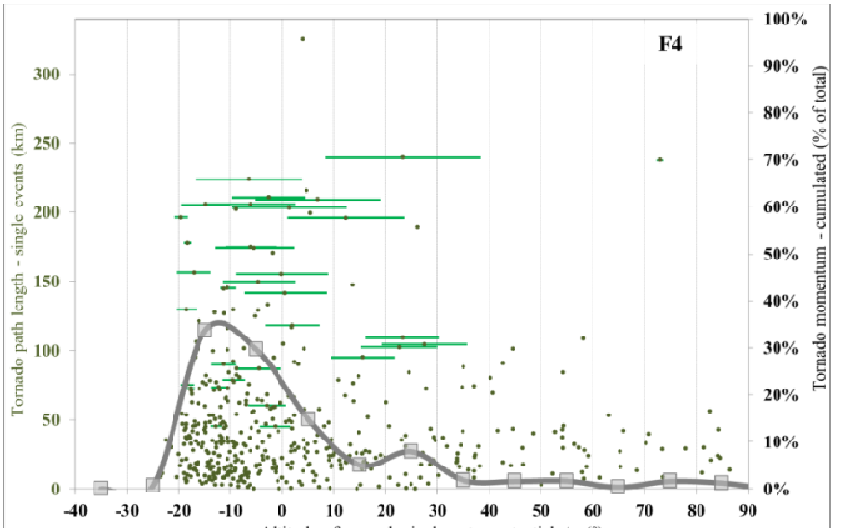

(d)

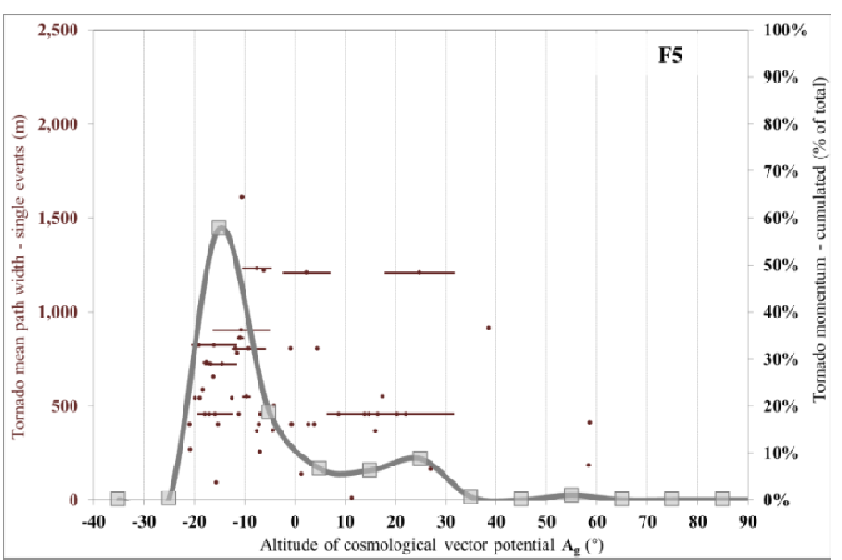

(e)

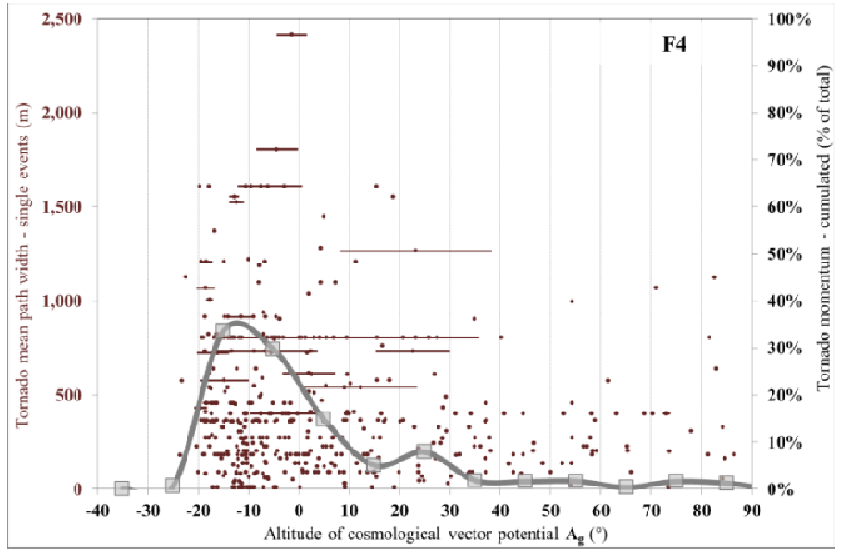

(f)

Fig. 7. Distribution of the overall tornado moment and moment of individual events $(a, b)$, path length $(c, d)$, mean path width $(e, f)$ for $F 5(a, c, e)$ and $F 4$ $(b, d, f)$ events, as functions of the altitude of the vector $A_{g}$.

The total moment for the F5 events shows a sharp distribution with a very high peak (about 60\%) in the interval $-20^{\circ}$ to $-10^{\circ}$, a substantial fraction between $-10^{\circ}$ and $10^{\circ}$ (about $18 \%$ ) and a secondary smaller peak around $20^{\circ}$ (about $8 \%$ ). No significant events are shown at $\mathbf{A}_{\mathbf{g}}$ altitudes greater than $30^{\circ}$. Moreover, looking at individual events (Fig. 7a), the vast majority of F5 tornadoes and especially the most energetic ones occur with $\mathrm{A}_{\mathrm{g}}$ altitudes between $-20^{\circ}$ and $-5^{\circ}$, with the biggest values between $-20^{\circ}$ and $-10^{\circ}$; while few 
relevant events occur with $\mathbf{A}_{\mathbf{g}}$ altitudes between $10^{\circ}$ and $25^{\circ}$ (always with some fraction of the lifetime spent with $\mathrm{A}_{\mathrm{g}}$ altitudes lower than $20^{\circ}$ ), a "dip" in the distribution of significant events arises approximately between $-5^{\circ}$ and $10^{\circ}$, all in all supporting not only the overall theoretical approach about the existence of a new energy source feeding violent tornadoes, but also the further hypothesis about the role of the vertical tilting of tornadoes described at the end of Section 3.2.

The above tentative conclusion is further confirmed by the analysis of the total moment of F4 events, which shows exactly the same qualitative features as for F5 events, but with a much less sharp and broader distribution, with the biggest peak reaching just 35\%, no "dip" of individual significant events arising around $0^{\circ}$ and few significant tornadoes occurring with $\mathrm{A}_{\mathrm{g}}$ altitudes as large as about $70^{\circ}$ (Fig. 7b). In other words, the intensity of tornadoes and therefore of the associated updraft looks like to activate a positive feedback with the energy feeding mechanisms proposed in Section 3.2.

Turning to the analysis of the tornado path length, which is indicative of the lifetime of the event, spans more than one order of magnitude and therefore contributes significantly to the overall tornado moment, the difference between F5 and F4 events is striking: practically all significant events (moment greater than $2 \cdot 10^{15} \mathrm{~J}$, as shown by the presence of the interval of $\mathrm{A}_{\mathrm{g}}$ altitudes) in the F5 class occur with $\mathbf{A}_{\mathbf{g}}$ altitudes in two intervals, i.e. between $-20^{\circ}$ and $-5^{\circ}$ and between $10^{\circ}$ and $25^{\circ}$, with much smaller path lengths at higher altitudes (Fig. 7c); conversely, the distribution of path lengths with $\mathbf{A}_{\mathbf{g}}$ altitudes is much broader and scattered when it comes to $\mathrm{F} 4$ events, which show few events with large path length even beyond $40^{\circ}$. Nevertheless, the majority of most energetic tornadoes still do show the largest path lengths between $-20^{\circ}$ and $20^{\circ}$ (Fig. $7 \mathrm{~d}$ ).

While spanning less than one order of magnitude, at least for the most energetic events, the mean path width still contributes significantly to the overall moment due to its connection with the tornado height, as explained in Section 2. Although the above considerations for the path length remain valid for the mean path width at least qualitatively, the charts are somehow less informative (Fig. 7e and Fig. 7f), likely due to the inherently greater uncertainty in the assessment of this quantity. Beyond the usual "bimodal" distribution around the altitude $0^{\circ}$, evident for the F5 events and missing for the F4 tornadoes, the most striking difference appears to be the absence of mean path widths larger than $500 \mathrm{~m}$ for the F5 events for $\mathrm{A}_{\mathrm{g}}$ altitudes exceeding $40^{\circ}$, while values greater than $500 \mathrm{~m}$ are still common up to the higher altitudes for F4 events.

All the above considerations appear in very good agreement with previous findings [8], as well as supportive of the hypothesized new mechanisms of energy supply to the most violent tornadoes by means of a positive feedback between updraft (and therefore rotation velocity) and the fundamental anisotropy of the physical space.

It should be noted however that the astronomical configuration doesn't help very much because it happens that the $\mathbf{A}_{\mathbf{g}}$ vector altitudes are around zero in the study area (as well as at any other site with the same latitude in the northern hemisphere) during the most favorable times of the day for the development of significant tornadoes, i.e. in the early to mid-afternoon, during the spring months, when solar heating is intense and growing, sea surface temperatures in the Gulf of Mexico are quickly rising and the jet stream flows still at sufficiently southern latitudes thus providing the needed vertical shear, moreover with residence time near $0^{\circ}$ decreasing northward, i.e. towards colder areas where violent tornadoes are less likely to occur.

As a consequence, an even more definitive proof of the theory described in this article could be achieved after performing a similar study elsewhere in the northern hemisphere, e.g. in Europe, and - more important - in the southern hemisphere where the vector $\mathbf{A}_{\mathbf{g}}$ is approximately reversed with regards to the horizontal plane, i.e. points downward during most of the time.

\section{Conclusions}

The main aim of this article was to detect the fingerprint of an hypothesized new energy source for violent tornadoes realized by means of a positive feedback between the tornado updraft and a fundamental anisotropy of the physical space.

The global anisotropy of the physical space was detected by means of a long series of experiments as well as observations of different natural systems at virtually any scale of the Universe, from the weak interaction to the metagalactic ones; on its basis the byuon theory (Section 3.1.) was built since the early 1990s', today allowing consistent explanations to a variety of physical phenomena covering the fields of nuclear physics, astrophysics, geophysics, as well as offering the chance to harness a new energy source $[17,18,20]$.

Following earlier research and in agreement with previous findings [8], this article extends the analysis of the hypothesized connection between the theory of byuons and the energy balance of the most violent tornadoes, which are likely the most powerful phenomena at scales from hundreds of meters to tenths of $\mathrm{km}$ in the Earth's atmosphere.

The additional convincing evidence provided in Section 4 on the basis of the largest available database makes even more difficult to dismantle the theoretical approach to the new source of energy sustaining the most powerful tornadoes.

As a consequence, it's possible to state that the most powerful tornadoes can occur only at specific times of the day which depend on latitude time of the year.

Nevertheless, as pointed out at the end of Section 4, although the herein presented evidence is convincing and agrees with the results and conclusions from previous research, further investigation is needed elsewhere in the northern hemisphere and, more important, in the southern hemisphere, in order to provide the really conclusive proof of the new hypothesized physical processes. 


\section{Acknowledgments}

The authors gratefully acknowledge M. Pagliaro for the outstanding support and motivation by means of the SuNEC 2013 Conference.

\section{Appendix}

Abbreviations used in this article:

$\mathrm{A}_{\Sigma}=$ Summary vector potential $(\mathrm{T} \cdot \mathrm{m})$

$\mathrm{A}_{\mathrm{g}}=$ Cosmological vector potential $(\mathrm{T} \cdot \mathrm{m})$

CST $=$ Central Standard Time

$\mathrm{TNT}=$ Trinitrotoluene

\section{References}

[1] S.A. Arsen'yev, Mathematical modeling of tornadoes and squall storms, Geosci. Front. 2 (2011) 215-221.

[2] S.A. Arsen'yev, N.K. Shelkovnikov, Electromagnetic fields in tornados and spouts, Moscow Univ. Phys. Bull. 67 (2012) 290-295.

[3] A.Y. Gubar, A.I. Avetisyan, V. V Babkova, Tornado rise: 3D numerical model in the mesoscale turbulence theory of Nikolaevskiy, Dokl. Earth Sci. 419 (2008) 467-472.

[4] N. Kufa, R. Snow, Lightning: meteorology's new tool, in: 86th AMS Annu. Meet. (Atlanta, GA). Second Conf. Meteorol. Appl. Light. Data, American Meteorological Society, 2006.

[5] A.J. Litta, U.C. Mohanty, S. Kiran Prasad, M. Mohapatra, A. Tyagi, S.C. Sahu, Simulation of tornado over Orissa (India) on March 31, 2009, using WRF-NMM model, Nat. Hazards. 61 (2011) 1219-1242.

[6] A.E. Mercer, C.M. Shafer, C.A. Doswell, L.M. Leslie, M.B. Richman, Objective Classification of Tornadic and Nontornadic Severe Weather Outbreaks, Mon. Weather Rev. 137 (2009) 4355-4368.

[7] L. Schielicke, P. Névir, Comprehensive analysis of tornado statistics in comparison to earthquakes: intensity and temporal behaviour, Nonlinear Process. Geophys. 20 (2013) 47-57.

[8] Y.A. Baurov, I.F. Malov, F. Meneguzzo, Tornadoes and the global anisotropy of the physical space, Am. J. Mod. Phys. 3 (2014) 93-112.

[9] SPC, Storm Prediction Center - severe weather database files (1950-2013), (2013).

[10] M. V Kurgansky, The statistical distribution of intense moistconvective, spiral vortices in the atmosphere, Dokl. Earth Sci. 371 (2000) 408-410.

[11] L. Schielicke, P. Névir, Introduction of an atmospheric moment combining Eulerian and Lagrangian aspects of vortices: Application to tornadoes, Atmos. Res. 100 (2011) 357-365.

[12] Y.A. Baurov, E.Y. Klimenko, S.I. Novikov, Experimental observation of space magnetic anisotropy, Phys. Lett. A. 162 (1992) 32-34.

[13] Y.A. Baurov, Space magnetic anisotropy and a new interaction in nature, Phys. Lett. A. 181 (1993) 283-288.

[14] Y.A. Baurov, A.A. Konradov, V.F. Kushniruk, E.A. Kuznetsov, Y.G. Sobolev, Y. V. Ryabov, et al., Experimental investigations of changes in beta-decay rate of 60Co and 137Cs, Mod. Phys. Lett. A. 16 (2001) 2089-2101.

[15] I.F. Malov, Y.A. Baurov, The distribution of space velocities of radio pulsars, Astron. Reports. 51 (2007) 830-835.

[16] Y.A. Baurov, The Anisotropy of Cosmic Rays and the Global Anisotropy of Physical Space, J. Mod. Phys. 03 (2012) 17441748.

[17] Y.A. Baurov, On the structure of physical vacuum and a new interaction in Nature (Theory, Experiment and Applications), Nova Science, NY, 2000.

[18] Y.A. Baurov, Global Anisotropy of Physical Space, Experimental and Theoretical Basis, Nova Science, NY, 2004.

[19] Y.A. Baurov, I.F. Malov, On the Nature of Dark Matter and Dark Energy, J. Mod. Phys. 01 (2010) 17-32.

[20] Y.A. Baurov, Research of global anisotropy of physical space based on investigation of changes in $\beta$ and $\alpha$-decay rate of radioactive elements, motion of pulsars and anisotropy of cosmic rays, Am. J. Mod. Phys. 2 (2013) 177-184.

[21] Y.A. Baurov, I.F. Malov, Variations of Decay Rates of Radioactive Elements and their Connections with Global Anisotropy of Physical Space, Int. J. Pure Appl. Phys. 6 (2010) 469-482. Also at: http://arxiv.org/abs/1001.5383.

[22] Y.A. Baurov, I.B. Timofeev, V.A. Chernikov, S.F. Chalkin, A.A. Konradov, Experimental investigations of the distribution of pulsed-plasma-generator radiation at its various spatial orientation and global anisotropy of space, Phys. Lett. A. 311 (2003) 512-523.

[23] Y.A. Baurov, Y.G. Sobolev, Y. V. Ryabov, V.F. Kushniruk, Experimental investigations of changes in the rate of beta decay of radioactive elements, Phys. At. Nucl. 70 (2007) $1825-1835$

[24] P.A. Sturrock, J.B. Buncher, E. Fischbach, D. Javorsek II, J.H. Jenkins, J.J. Mattes, Concerning the Phases of the Annual Variations of Nuclear Decay Rates, Astrophys. J. 737 (2011) 65 .

[25] Y.A. Baurov, A.A. Spitalnaya, A.A. Abramayan, V.A. Solodovnikov, Seismic activity of the earth, the cosmological vectorial potential and method of a short-term earthquakes forecasting, Nat. Sci. 03 (2011) 109-119.

[26] Y.A. Baurov, F. Meneguzzo, A.Y. Baurov, A.Y.J. Baurov, Plasma Vacuum Bubbles and a New Force of Nature, The Experiments, Int. J. Pure Appl. Sci. Technol. 11 (2012) 34-44.

[27] Y.A. Baurov, L. Albanese, F. Meneguzzo, New force and new heat, Am. J. Astron. Astrophys. (in press) (2014) Special Issue: Global Anisotropy, Theory of Byuon. 